\title{
On Constructing Minimal Formulae
}

\author{
Paul E. Dunne \\ Dept. of Computer Science, The University of Liverpool, Liverpool, United Kingdom
}

\begin{abstract}
Given a Boolean propositional formula, $\varphi\left(X_{n}\right)$ over the basis $\Omega=\{\wedge, \vee, \neg\}$ we consider the following decision problem: is there a subset of literals, $S$, for which $\varphi\left(X_{n}\right) \equiv \bigwedge_{y \in S} y$ or $\varphi\left(X_{n}\right) \equiv \bigvee_{y \in S} y$ ? We prove that the "obvious" $\Sigma_{2}^{p}$ upper bound is sub-optimal and that the problem is decidable in $\mathrm{P}_{\|}^{\mathrm{NP}}$ the class of languages decidable by polynomial time methods allowed to make non-adaptive queries to an NP oracle. We further show that the associated function problem of computing a witnessing such subset when one exists can be solved in $\mathrm{FP}_{\|}^{\mathrm{NP}}$.
\end{abstract}

Key words:

\section{Introduction}

A long-standing problem in computational complexity theory concerns how difficult it is to determine whether a given propositional formula, $\varphi\left(X_{n}\right)$, can be rewritten as an equivalent formula, $\psi\left(X_{n}\right)$, whose size is strictly smaller. Here the size of a formula is interpreted as the number of occurences of literals $-x$ and $\neg x$ - in the formula's specification. This problem was studied by Hemaspaandra and Wechsung $[11,12]$ who obtained the first non-trivial lower bounds on its complexity. These establish that, in its most general form, Minimal Expression Equivalence (MEE) is $\mathrm{P}_{\|}^{\mathrm{NP}}$-hard. To date, however, no improvement to the obvious $\Sigma_{2}^{p}$ upper bound has been obtained, i.e. the approach which given an instance $\langle\varphi, k\rangle$ of MEE, 
proceeds by guessing a formula $\psi$ of size at most $k$ and accepts if and only if $\varphi \equiv \psi$

In this note, although we do not obtain improved bounds for the general MEE problem, we are able to show that a related problem, in which the structure of witnessing equivalent formulae is very tightly constrained, can be decided in $\mathrm{P}_{\|}^{\mathrm{NP}}$. Furthermore our proof of this upper bound immediately yields an algorithm for constructing such a set: this algorithm places the related search problem in the function class $\mathrm{FP}_{\|}^{\mathrm{NP}}$. The exact variant of MEE we consider will be called Term Equivalence (TE) and is defined as follows:

Term Equivalence (TE)

Instance: $\varphi\left(X_{n}\right)$ propositional formula over variables $\left\{x_{1}, \ldots, x_{n}\right\}$ and logical operations $\{\wedge, \vee, \neg\}$.

Question: $\exists S \subset\left\{x_{1}, \ldots, x_{n}, \neg x_{1}, \ldots, \neg x_{n}\right\}$ such that either

$$
\varphi\left(X_{n}\right) \equiv\left(\bigwedge_{y \in S} y\right) \quad \text { or } \varphi\left(X_{n}\right) \equiv\left(\bigvee_{y \in S} y\right) ?
$$

We denote by FTE the associated function problem, i.e.

$$
\operatorname{FTE}\left(\varphi\left(X_{n}\right)\right)= \begin{cases}\text { undefined } & \text { if } \varphi \notin \mathrm{TE} \\ \min S & \text { if } \varphi \equiv \wedge_{y \in S} y \text { or } \varphi \equiv \vee_{y \in S} y\end{cases}
$$

Here min is with respect to $\subseteq$ and thus covers the cases where $\varphi$ is equivalent to a Boolean constant function so that $S=\emptyset$ is reported if $\varphi \equiv \top \equiv \wedge_{y \in S} y$ or $\varphi \equiv \perp \equiv \vee_{y \in S} y$.

The upper bound methods combine a translation from formulae to directed graph structures with recent complexity results on identifying subsets of vertices satisfying specific criteria from Dunne [7,8]. The study of such sets originates from the concept of so-called "extension semantics" in the argument systems pioneered in work of Dung [3]. Readers interested in a general overview of these 
and related systems are referred to the survey of Bench-Capon and Dunne [1]; a detailed introduction to complexity-theoretic work in this model is provided in Dunne and Wooldridge [10].

In the remainder of this note we present background definitions including the graph-theoretic terminology subsequently used in Section 2. Section 3 describes the translation from formulae over the basis $\{\wedge, \vee, \neg\}$ to directed graphs that are referred to as formula graphs. Our main result is presented in Section 4 with conclusions given in Section 5.

\section{Background Definitions}

A propositional formula, $\varphi\left(X_{n}\right)$ where $X_{n}=\left\{x_{1}, \ldots, x_{n}\right\}$ is a set of $n$ Boolean variables is any structure built according to the following rules.

a. A literal $y \in\left\{x_{1}, x_{2}, \ldots, x_{n}, \neg x_{1}, \ldots, \neg x_{n}\right\}$ is an $\wedge$-formula and also an $\checkmark$-formula.

b. If $\left\{\varphi_{1}, \ldots, \varphi_{k}\right\}$ are all $\vee$-formulae then $\left(\varphi_{1}\right) \wedge\left(\varphi_{2}\right) \cdots \wedge\left(\varphi_{k}\right)$ is an $\wedge$-formula.

c. If $\left\{\varphi_{1}, \ldots, \varphi_{k}\right\}$ are all $\wedge$-formulae then $\left(\varphi_{1}\right) \vee\left(\varphi_{2}\right) \cdots \vee\left(\varphi_{k}\right)$ is an $\vee$-formula.

d. $\psi$ is a $\{\wedge, \vee, \neg\}$-formula (or more simply just formula) if $\psi$ is the result of a finite number of applications of (a)-(c) above.

We say an $\wedge$-formula is an elementary conjunction if it has the from $\wedge_{y \in S} y$ for some subset $S$ of $\left\{x_{1}, x_{2}, \ldots, x_{n}, \neg x_{1}, \ldots, \neg x_{n}\right\}$ such that at most one of $x_{i}, \neg x_{i}$ belongs to $S(1 \leq i \leq n)$. Similarly an $\vee$-formula is an elementary disjunction if it is of the form $\vee_{y \in S} y$ (where again $S$ does not contain both a literal and its negation).

We note a number of points concerning this definition. Firstly we do not restrict the operations $\wedge$ and an $\vee$ to be purely binary. Secondly we do not explicitly allow the constant symbols $\perp$ (false) or $\top$ (true) as formulae. 
Suppose $\pi=\left\langle p_{1}, \ldots, p_{n}\right\rangle \in\langle\perp, \top, *\rangle^{n}$ is a partial assignment of Boolean values to the propositional variables $\left\langle x_{1}, \ldots, x_{n}\right\rangle$ defining some formula $\varphi\left(x_{1}, \ldots, x_{n}\right)$. The value $\varphi(\alpha) \in\{\perp, \top, *\}$ is recursively defined via:

1. If $\varphi\left(X_{n}\right)$ is the literal $x_{i}$ then $\varphi(\pi)=p_{i}$.

2. If $\varphi\left(X_{n}\right)$ is the literal $\neg x_{i}$ then $\varphi(\pi)=\neg p_{i}$ if $p_{i} \neq *$ and $*$ otherwise, i.e. $\top$ if $p_{i}=\perp ; \perp$ if $p_{i}=\top$.

3. If $\varphi\left(X_{n}\right)$ is an $\wedge$-formula formed from $\vee$-formulae $\left\{\varphi_{1}, \ldots, \varphi_{k}\right\}$ then:

a. $\varphi(\pi)=\top$ if $\varphi_{j}(\pi)=\top$ for all $1 \leq j \leq k$.

b. $\varphi(\pi)=\perp$ if $\varphi_{j}(\pi)=\perp$ for some $1 \leq j \leq k$.

c. $\varphi(\pi)=*$ otherwise, i.e no $\varphi_{j}(\pi)=\perp$ and at least one $\varphi_{j}(\pi)=*$.

4. If $\varphi\left(X_{n}\right)$ is an $\vee$-formula formed from $\wedge$-formulae $\left\{\varphi_{1}, \ldots, \varphi_{k}\right\}$ then:

a. $\varphi(\pi)=\perp$ if $\varphi_{j}(\pi)=\perp$ for all $1 \leq j \leq k$.

b. $\varphi(\pi)=\top$ if $\varphi_{j}(\pi)=\top$ for some $1 \leq j \leq k$.

c. $\varphi(\pi)=*$ otherwise, i.e no $\varphi_{j}(\pi)=\top$ and at least one $\varphi_{j}(\pi)=*$.

Two formulae, $\varphi\left(X_{n}\right)$ and $\psi\left(X_{n}\right)$ are logically equivalent - denoted $\varphi\left(X_{n}\right) \equiv$ $\psi\left(X_{n}\right)$ - if for all assignments $\alpha \in\langle\perp, \top\rangle^{n}$ it holds that $\varphi(\alpha)=\psi(\alpha)$. We note that logical equivalence is well-defined in the case when the propositional variables, $Y$, defining $\psi$ are a strict subset of those defining $\varphi$ : in this case $\varphi(X) \equiv$ $\psi(Y)$ if for every assignment $\alpha$ of $X, \varphi(\alpha)=\psi(\beta)$ where $\beta$ is the projection of the assignment $\alpha$ onto the variables $Y \subset X$.

Through the normal forms conjunctive normal form (CNF) and disjunctive normal form (DNF) it well known that given any Boolean function $f:\langle\perp, \top\rangle^{n} \rightarrow$ $\langle\perp, \top\rangle$ there is an $\wedge$-formula, $\psi_{\wedge}$ and an $\vee$-formula $\psi_{\vee}$ for which $\psi_{\wedge}\left(X_{n}\right) \equiv$ $\psi_{\vee}\left(X_{n}\right) \equiv f\left(X_{n}\right)$, i.e. $\forall \alpha \in\langle\perp, \top\rangle^{n} \psi_{\wedge}(\alpha)=\psi_{\vee}(\alpha)=f(\alpha)$, see, e.g. [5, pp. 12-13]. 
The size of a formula $\varphi\left(X_{n}\right)$ (denoted $\left|\varphi\left(X_{n}\right)\right|$ ) is the number of occurences of literals used in defining it, i.e

$$
\left|\varphi\left(X_{n}\right)\right|=\left\{\begin{array}{lll}
1 & \text { if } & \varphi\left(X_{n}\right)=y \text { for some literal } y \\
\sum_{i=1}^{k}\left|\varphi_{i}\left(X_{n}\right)\right| & \text { if } & \varphi\left(X_{n}\right) \text { is an } \wedge \text {-formula formed from }\left\{\varphi_{1}, \ldots, \varphi_{k}\right\} \\
\sum_{i=1}^{k}\left|\varphi_{i}\left(X_{n}\right)\right| & \text { if } & \varphi\left(X_{n}\right) \text { is an } \vee \text {-formula formed from }\left\{\varphi_{1}, \ldots, \varphi_{k}\right\}
\end{array}\right.
$$

We next introduce a number of graph-theoretic concepts which will be important in our translation of formulae to directed graphs and in the upper bound arguments of Section 4. Throughout the sequel, $H(V, F)$ is a directed graph with vertices $V=\left\{v_{1}, \ldots, v_{n}\right\}$ and edges $F \subseteq V \times V$. For $S \subseteq V$ the sets $S^{-}$and $S^{+}$ are

$$
\begin{aligned}
& S^{-}=\{w: \exists v \in S \text { for which }\langle w, v\rangle \in F\} \\
& S^{+}=\{w: \exists v \in S \text { for which }\langle v, w\rangle \in F\}
\end{aligned}
$$

It should be noted that we permit occurences of "self-loops" in $F$, i.e. we allow $\langle v, v\rangle \in F^{1}$

A subset $S$ of $V$ is conflict-free if $F$ contains no edges in $S \times S$. The subset $S$ is admissible if it is both conflict-free and for every $v \in S^{-},\{v\}^{-} \cap S \neq \emptyset$. An admissible set is preferred if it is maximal w.r.t. $\subseteq$, i.e. if $S$ is preferred then for all $T \subseteq V$ if $S \subset T$ then $T$ is not admissible. A subset $S$ is ideal if $S$ is both admissible and a subset of every preferred set. ${ }^{2}$

We note the following results concerning these:

Fact 1. Given $H(V, F)$ and $S \subseteq V$,

\footnotetext{
${ }^{1}$ This is solely in order to simplify some of the subsequent technical lemmata. All of our results, albeit with rather more involved constructions, can be derived when self-loops are forbidden.

${ }^{2}$ Our choice of terminology coincides with the treatment of these concepts in the context of argument systems: it is, of course, the case that a number of these ideas are already well-established in graph-theoretic treaments, e.g. "conflict-free" corresponds with "independent set".
} 
a. Deciding if $S$ is admissible is in $\mathrm{P}$.

b. Deciding if $S$ is preferred is coNP-complete, [2].

c. Given $v \in V$ deciding if $v$ is a member of at least one preferred set is NPcomplete, [2].

d. Given $v \in V$ deciding if $v$ is a member of every preferred set is $\Pi_{2}^{p}$-complete. [9].

e. Deciding if $S$ is ideal is coNP-complete. [7, 8].

f. Every $H(V, F)$ has a unique maximal (w.r.t. $\subseteq$ ) ideal set. [4].

g. Deciding if $S$ is the maximal ideal set is $\mathrm{P}_{\|}^{\mathrm{NP}}$-complete, where hardness is with respect to randomized reductions that succeed with probability $1-2^{-|V|},[7$, 8].

h. Computing the maximal ideal set is $\mathrm{FP}_{\|}^{\mathrm{NP}}$-complete, [7, 8]. We note that the hardness proof uses a deterministic reduction.

The techniques used to establish Fact 1(d) and (h) play a significant role in our subsequent technical development.

\section{Formula Graphs and their Properties}

We now present a translation from formulae, $\varphi\left(X_{n}\right)$ as defined in Section 2 to directed graphs, $H_{\varphi}\left(V_{\varphi}, F_{\varphi}\right)$ and reprise some properties of admissible and preferred sets of vertices in $H_{\varphi}$. Our translation while similar to the standard representation of formulae as directed graphs, e.g. as described in [5, pp. 18-23], has some important differences. The original definition of formula graph as given in Defn. 2 was presented in [9, Defn. 7, p. 193].

We start with the well-known translation from formulae $\varphi\left(X_{n}\right)$ to trees.

Definition 1. Let $\varphi\left(X_{n}\right)$ be a propositional formula over the variables $X_{n}=$ $\left\langle x_{1}, x_{2}, \ldots, x_{n}\right\rangle$ using the operations $\{\wedge, \vee, \neg\}$ with negation applied only to vari- 


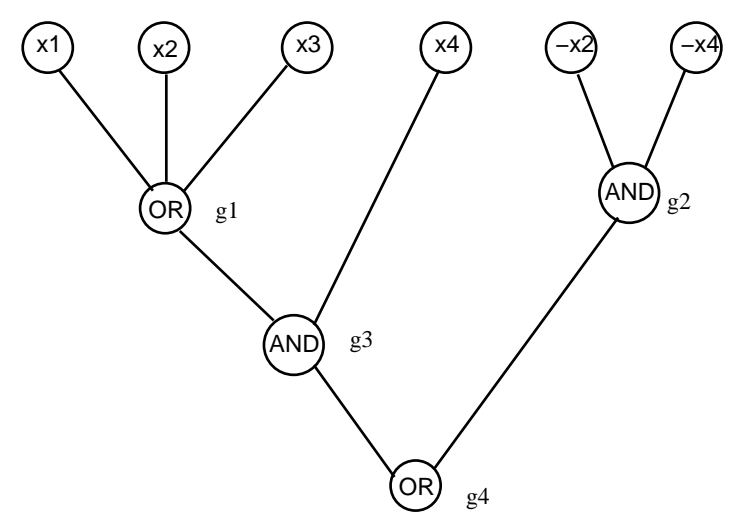

Figure 1: $T_{\varphi}\left(x_{1}, x_{2}, x_{3}, x_{4}\right)$ for $\left(\left(x_{1} \vee x_{2} \vee x_{3}\right) \wedge\left(x_{4}\right)\right) \vee\left(\neg x_{2} \wedge \neg x_{4}\right)$

ables of $\varphi$. The tree representation of $\varphi$ (denoted $\left.T_{\varphi}\right)$ is a rooted directed tree with root vertex denoted $\rho\left(T_{\varphi}\right)$ and inductively defined by the following rules.

a. If $\varphi\left(X_{n}\right)=y-a$ single literal $x$ or $\neg x-$ then $T_{\varphi}$ consists of a single vertex $\rho\left(T_{\varphi}\right)$ labelled $y$.

b. If $\varphi\left(X_{n}\right)$ is an $\wedge$-formula $\wedge_{i=1}^{k} \psi_{i}\left(X_{n}\right)$, with $\left\langle\psi_{1}, \psi_{2}, \ldots, \psi_{k}\right\rangle \vee$-formulae, then $T_{\varphi}$ is formed from the $k$ tree representations $\left\langle T_{\psi_{i}}\right\rangle$ by directing edges from each $\rho\left(T_{\psi_{i}}\right)$ into a new root vertex $\rho\left(T_{\varphi}\right)$ labelled $\wedge$.

c. If $\varphi\left(X_{n}\right)$ is an $\vee$-formula $=\vee_{i=1}^{k} \psi_{i}\left(X_{n}\right)$, with $\left\langle\psi_{1}, \psi_{2}, \ldots, \psi_{k}\right\rangle \wedge$-formulae, then $T_{\varphi}$ is formed from the $k$ tree representations $\left\langle T_{\psi_{i}}\right\rangle$ by directing edges from each $\rho\left(T_{\psi_{i}}\right)$ into a new root vertex $\rho\left(T_{\varphi}\right)$ labelled $\vee$.

In what follows we use the term node of $T_{\varphi}$ to refer to an arbitrary tree vertex, i.e. a leaf or internal vertex.

In the tree representation of $\varphi$, each leaf vertex is labelled with some literal $w$, (several leaves may be labelled with the same literal), and each internal vertex with an operation in $\{\wedge, \vee\}$. We shall subsequently refer to the internal vertices of $T_{\varphi}$ as the gates of the tree. Notice that the definition of formula ensures that the 
successor of any $\wedge$-gate (tree vertex labelled $\wedge$ ) is an $\vee$-gate (tree vertex labelled $\vee)$ and vice-versa. Let $m$ be the number of gate nodes in $T_{\varphi}$ and denote these gates by $\left\langle g_{1}, g_{2}, \ldots, g_{m}\right\rangle$ with $g_{m}$ taken as the root $\rho\left(T_{\varphi}\right)$ of the tree. For any edge $\langle h, g\rangle$ in $T_{\varphi}$ we refer to the node $h$ as an input of the gate $g$.

The directed graph structure used in our upper bound proof is obtained from the formula graph of $\varphi\left(X_{n}\right)$ as defined below.

Definition 2. Let $\varphi\left(X_{n}\right)$ be a formula with tree representation $T_{\varphi}$ having $m$ gates. The Formula Graph of $\varphi$, is the directed graph $H_{\varphi}\left(V_{\varphi}, F_{\varphi}\right)$ defined as follows.

$V_{\varphi}$ contains the following vertices

$X 12 n$ vertices representing literals. $\left\{x_{i}, \neg x_{i}: 1 \leq i \leq n\right\}$.

$X 2$ For each gate $g_{k}$ of $T_{\varphi}$, a vertex labelled $\neg g_{k}$ (if $g_{k}$ is an $\vee$-gate) or a vertex labelled $g_{k}$ (if $g_{k}$ is an $\wedge$-gate). If $g_{m}$, i.e the root of $T_{\varphi}$, happens to be an $\vee$-gate, then an additional vertex $g_{m+1}$ is included. We subsequently denote the set of vertices contributed by gates of $T_{\varphi}$ as $\mathcal{G}_{\varphi}$.

The directed edges $-F_{\varphi}-$ over $V_{\varphi}$ are

F1 $\left\{\left\langle x_{i}, \neg x_{i}\right\rangle,\left\langle\neg x_{i}, x_{i}\right\rangle: 1 \leq i \leq n\right\}$

F2 $\left\langle\neg g_{m}, g_{m+1}\right\rangle$ if $g_{m}$ is an $\vee$-gate in $T_{\varphi}$,

F3 If $g_{k}$ is an $\wedge$-gate in $T_{\varphi}$ with inputs $\left\{h_{1}, h_{2}, \ldots, h_{r}\right\}:\left\{\left\langle\neg h_{i}, g_{k}\right\rangle: 1 \leq i \leq\right.$ $r\}$.

It should be noted that when a literal vertex, $y$, is an input to an $\wedge$-gate in $T_{\varphi}$ then this convention leads to the corresponding edge originating from the literal vertex $\neg$ of $V_{\varphi}$.

F4 If $g_{k}$ is an $\vee$-gate in $T_{\varphi}$ with inputs $\left\{h_{1}, h_{2}, \ldots, h_{r}\right\}:\left\{\left\langle h_{i}, \neg g_{k}\right\rangle: 1 \leq i \leq\right.$ $r\}$. 


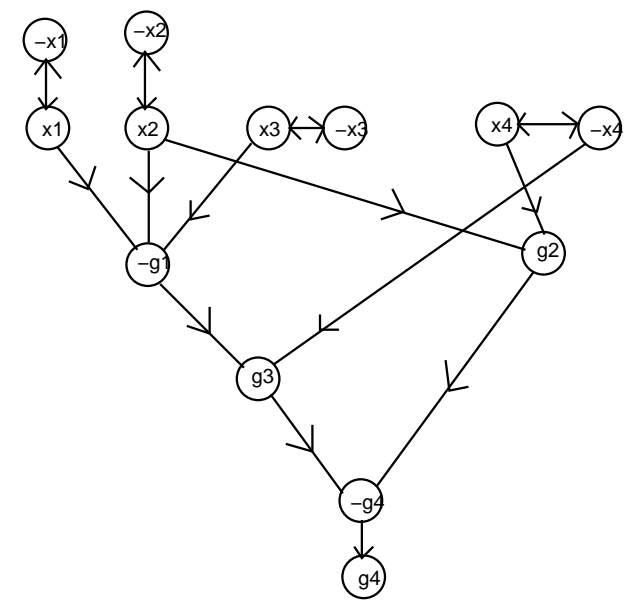

Figure 2: The Formula Graph $H_{\varphi}$ from the tree representation of Fig. 1

Fig. 2 shows the result of this translation when it is applied to the tree representation of the formula in Fig. 1.

Suppose $\pi: X_{n} \rightarrow\langle\perp, \top, *\rangle^{n}$ is a partial assignment of Boolean values to $X_{n}$. Any such assignment induces a partition of $V_{\varphi}$ which we denote

$$
\tau: V_{\varphi} \times\langle\perp, \top, *\rangle \rightarrow\{\text { In, Out, Open }\}
$$

The mapping $\tau(h, \pi)$ is given by,

$$
\tau(h, \pi)= \begin{cases}\text { In } \quad \text { if } \quad & h=x_{i} \text { and } \pi\left(x_{i}\right)=\top \text { or } \\ & h=\neg x_{i} \text { and } \pi\left(x_{i}\right)=\perp \text { or } \\ & h \text { is a gate with inputs }\left\{h_{1}, \ldots, h_{r}\right\} \\ & \text { and } \forall 1 \leq i \leq r \tau\left(h_{i}, \pi\right)=\text { Out } \\ \text { Out } \quad \text { if } \quad & h=x_{i} \text { and } \pi\left(x_{i}\right)=\perp \text { or } \\ & h=\neg x_{i} \text { and } \pi\left(x_{i}\right)=\top \text { or } \\ & h \text { is a gate with inputs }\left\{h_{1}, \ldots, h_{r}\right\} \\ & \text { and } \exists 1 \leq i \leq r \tau\left(h_{i}, \pi\right)=I n \\ & \text { otherwise }\end{cases}
$$


For example, with respect to the formula graph shown in Fig. 2 which resulted from the $\vee$-formula $\left(\left(x_{1} \vee x_{2} \vee x_{3}\right) \wedge\left(x_{4}\right)\right) \vee\left(\neg x_{2} \wedge \neg x_{4}\right)$, if $\pi=\langle\top, *, *, \top\rangle$ then $\left\{x_{1}, x_{4}, g_{3}, g_{4}\right\}$ are all labelled In by $\tau ;\left\{\neg x_{1}, \neg x_{4}, \neg g_{1}, g_{2}, \neg g_{4}\right\}$ are labelled Out and all other nodes are labellel Open. For $\beta=\langle *, \perp, *, \perp\rangle,\left\{\neg x_{2}, \neg x_{4}, g_{2}, g_{4}\right\}$ are all labelled $I n$ and $\left\{x_{2}, x_{4}, g_{3}, \neg g_{4}\right\}$ labelled Out.

Let $\operatorname{In}_{\varphi}(\pi)=\left\{h \in V_{\varphi}: \tau(h, \pi)=\operatorname{In}\right\}$.

\section{Theorem 1.}

a. For all $H\left(V_{\varphi}, F_{\varphi}\right)$ and all partial assignments $\pi$ the set $\operatorname{In}_{\varphi}(\pi)$ is admissible.

b. For $S \subseteq V_{\varphi}, S$ is preferred if and only if $S=\operatorname{In}_{\varphi}(\alpha)$ for some total assignment $\alpha$, i.e. $\alpha\left(x_{i}\right) \in\{\perp, \top\}$ for every $x_{i}$.

Proof: For (a), if $\operatorname{In}_{\varphi}(\pi)=\emptyset$ then we are done since the empty set is always admissible. Otherwise $\operatorname{In}_{\varphi}(\pi)$ contains a non-empty subset of $V_{\varphi}$. This set must be conflict-free since, by the definition of $\tau(h, \pi)$ if $\tau(h, \pi)=\operatorname{In}$ then $\tau(p, \pi) \neq$ In for any node $p$ which is adjacent to $h$ in $H_{\varphi}$ : if $\langle h, p\rangle \in F_{\varphi}$ then $p$ is either a literal $(\neg h)$ or a gate with $h$ as an input; if $\langle p, h\rangle \in F_{\varphi}$ either $h$ is the literal $\neg p$ or $h$ is a gate with $p$ as an input. In all cases $p \in \operatorname{In}(\pi)$ would preclude $h \in \operatorname{In}(\pi)$. Now consider any $h$ in $\left\{\operatorname{In}_{\varphi}(\pi)\right\}^{-}$. By definition, $\tau(h, \pi)=$ Out. But $\tau(h, \pi)=$ Out only if $h$ is a literal with $\tau(\neg h, \pi)=I n$ or if $h$ is a gate vertex one of whose inputs is in $\operatorname{In}_{\varphi}(\pi)$ : in both cases $\operatorname{In}_{\varphi}(\pi) \cap\{h\}^{-} \neq \emptyset$ so that $\operatorname{In}_{\varphi}(\pi)$ is admissible.

For (b), first note that if $\alpha$ is a total assignment then $\tau(h, \alpha) \in\{$ In, Out $\}$ for all $h \in V_{\varphi}$. From (a) we know that $\operatorname{In}_{\varphi}(\alpha)$ is admissible. To see that it is a maximal such set it suffices to note that $\tau(h, \alpha)=$ Out implies there is some $g \in \operatorname{In}_{\varphi}(\alpha)$ for which $\langle g, h\rangle \in F_{\varphi}$, i.e. $\{h\} \cup \operatorname{In}_{\varphi}(\alpha)$ would not be conflict-free and, hence, is not admissible. Conversely, if $S \subseteq V_{\varphi}$ is preferred then $S$ must contain exactly one literal vertex from each of the $n$ pairs $\left\{x_{i}, \neg x_{i}\right\}$. Defining the 
total assignment $\alpha_{S}$ by $\alpha_{S}\left(x_{i}\right)=\top$ if $x_{i} \in S$; $\alpha_{S}\left(x_{i}\right)=\perp$ if $\neg x_{i} \in S$, it is immediate that $S=\operatorname{In}_{\varphi}\left(\alpha_{S}\right)$.

The partition induced by a partial assignment $\pi$ has a close relationship to the value in $\{\perp, \top, *\}$ taken by nodes, i.e. literals and gates, in the tree representation of a formula. We recall that value $(h, \pi)$ for $h$ a node in the tree representation of a formula and $\pi=\left\langle p_{1}, \ldots, p_{n}\right\rangle \in\langle\perp, \top, *\rangle^{n}$ a partial assignment of $X_{n}$, is defined $\mathrm{via}^{3}$

$$
\operatorname{value}(h, \pi)=\left\{\begin{array}{lll}
\top & \text { if } \quad h=x_{i} \text { and } p_{i}=\top \text { or } \\
& \\
& h=\neg x_{i} \text { and } p_{i}=\perp \\
& \text { if } \quad h=x_{i} \text { and } p_{i}=\perp \text { or } \\
& & h=\neg x_{i} \text { and } p_{i}=\top \\
& \text { if } \quad h=x_{i} \text { and } p_{i}=* \text { or } \\
& & h=\neg x_{i} \text { and } p_{i}=* \\
& \text { if } \quad h \text { is an } \wedge \text {-gate with inputs }\left\{h_{1}, \ldots, h_{r}\right\} \\
\bigwedge_{i=1}^{r} \operatorname{value}\left(h_{i}, \pi\right) & \operatorname{value}\left(h_{i}, \pi\right) & \text { if } \quad h \text { is an } \vee \text {-gate with inputs }\left\{h_{1}, \ldots, h_{r}\right\}
\end{array}\right.
$$

Theorem 2. Let $\varphi\left(X_{n}\right)$ be a formula with tree representation $T_{\varphi}$ and formula graph $H_{\varphi}\left(V_{\varphi}, F_{\varphi}\right)$. For $h$ a vertex of the formula graph - i.e. node of the tree representation - and $\pi$ any partial assignment:

\footnotetext{
${ }^{3}$ The value of $\wedge($ resp. $\vee)$ w.r.t partial assignments is given in the obvious way by $\wedge_{i=1}^{k} y_{i}$ is $\top$ (if $y_{i}=\top$ for all $1 \leq i \leq k$ ); $\perp$ (if at least one $y_{i}=\perp$ ); and $*$ in all other cases. Similarly $\vee_{i=1}^{k} y_{i}$ is $\perp$ (if $y_{i}=\perp$ for all $1 \leq i \leq k$ ); $\top$ (if at least one $y_{i}=\top$ ); and $*$ in all other cases.
} 


$$
\tau(h, \pi)=\operatorname{In}(\text { resp. } \tau(h, \pi)=\text { Out) if and only if }
$$

$$
\left\{\begin{array}{rrl}
h \text { is a literal vertex } & \text { and } & \operatorname{value}(h, \pi)=\top \\
\text { resp. } & \operatorname{value}(h, \pi)=\perp \\
h \text { is an } \wedge \text {-gate } & \text { and } & \operatorname{value}(h, \pi)=\top \\
\text { resp. } & \operatorname{value}(h, \pi)=\perp \\
h \text { is an } \vee \text {-gate } & \text { and } & \operatorname{value}(h, \pi)=\perp \\
\text { (labelled } \left.\neg h \text { in } H_{\varphi}\right) & \text { resp. } & \operatorname{value}(h, \pi)=\top
\end{array}\right.
$$

Similarly $\tau(h, \pi)=$ Open if and only if value $(h, \pi)=*$.

Proof: It is easily checked that $\tau(h, \pi)=$ Open if and only if value $(h, \pi)=*$. For the remaining cases, we recall that the depth of a node (in either representation) has $\operatorname{depth}(h)=0$ if $h$ is a literal node and

$$
1+\max \left\{\operatorname{depth}\left(h_{i}\right): h \text { is a gate node with inputs }\left\{h_{1}, \ldots, h_{r}\right\}\right\}
$$

when $h$ is a gate node

We proceed by induction on the depth of nodes.

For the inductive base $\operatorname{depth}(h)=0$, the only relevant nodes are literals and the definitions of $\tau(y, \pi)$ and value $(y, \pi)$ immediately give

$$
\begin{gathered}
\tau(y, \pi)=\text { In } \Leftrightarrow \operatorname{value}(y, \pi)=\top \\
\tau(y, \pi)=\text { Out } \Leftrightarrow \operatorname{value}(y, \pi)=\perp
\end{gathered}
$$

Assuming the theorem holds for all nodes at depth less than $k \geq 1$ we show it holds for all nodes whose depth is equal to $k$. Let $h$ be any node with $\operatorname{depth}(h)=k \geq 1$. Then $h$ must be a gate node with inputs $\left\{h_{1}, \ldots, h_{r}\right\}$. Furthermore, $\operatorname{depth}\left(h_{i}\right)<k$ for each input $h_{i}$ of $h$.

Suppose first that $h$ is an $\wedge$-gate and $\tau(h, \pi)=$ In. Each $h_{i}$ must have $\tau\left(h_{i}, \pi\right)=O u t$, and thus by the inductive hypothesis, if $h_{i}$ is an $\vee$-gate, value $\left(h_{i}, \pi\right)=$ 
$\top$ and if $h_{i}$ is the literal $y$ then value $(y, \pi)=\perp$, i.e. value $(\neg y, \pi)=\top$. From the definition of formula graph if $h$ is an $\wedge$-gate one of whose inputs (in $T_{\varphi}$ ) is the literal $y$, then the edge directed into $h$ (in $H_{\varphi}$ ) is $\langle\neg y, h\rangle$. In other words,

$$
\begin{gathered}
\langle y, h\rangle \in F_{\varphi} \text { and }(h \text { is an } \wedge \text {-gate }) \text { and } \tau(h, \pi)=I n \\
\text { implies }
\end{gathered}
$$

$$
\langle\neg y, h\rangle \in T_{\varphi} \text { and } \tau(y, \pi)=O u t
$$

In summary from $h$ an $\wedge$-gate with $\tau(h, \pi)=I n$ we have $\tau\left(h_{i}, \pi\right)=O u t$ for all inputs $h_{i}$, hence value $\left(h_{i}, \pi\right)=\top$ (from relation (1) and the inductive hypothesis) so that value $(h, \pi)=\wedge_{i=1}^{r} \operatorname{value}\left(h_{i}, \pi\right)=\top$. On the other hand, if $\tau(h, \pi)=$ Out then there must be some input $h_{i}$ for which $\tau\left(h_{i}, \pi\right)=I n$. If $h_{i}$ is the literal $\neg y$ we have $\operatorname{value}(y, \pi)=\perp$ giving $\operatorname{value}(h, \pi)=\perp$ as required. If $h_{i}$ is an $\vee$-gate with $\tau\left(h_{i}, \pi\right)=I n$ then from the inductive hypothesis, value $\left(h_{i}, \pi\right)=\perp$ and again value $(h, \pi)=\perp$.

Now suppose $h$ is an $\vee$-gate with inputs $\left\{h_{1}, \ldots, h_{r}\right\}$ and $\tau(h, \pi)=I n$. We again have $\tau\left(h_{i}, \pi\right)=$ Out so that from the inductive hypothesis (and the fact that inputs are either $\wedge$-formulae or literals), value $\left(h_{i}, \pi\right)=\perp$ leading to $\operatorname{value}(h, \pi)=\vee_{i=1}^{k} \operatorname{value}\left(h_{i}, \pi\right)=\perp$ as required. Similarly is $\tau(h, \pi)=$ Out then some input must have $\tau\left(h_{i}, \pi\right)=I n$ and again (via the inductive hypothesis) $\operatorname{value}\left(h_{i}, \pi\right)=\top$ leading to $\operatorname{value}(h, \pi)=\vee_{i=1}^{r} \operatorname{value}\left(h_{i}, \pi\right)=\top$.

For the converse direction, the inductive base $(\operatorname{depth}(h)=0)$ has already been established. Thus suppose $h$ is a gate node with $\operatorname{value}(h, \pi)=\top$. If $h$ is an $\wedge$-gate we need to show $\tau(h, \pi)=I n$. Letting $\left\{h_{1}, \ldots, h_{r}\right\}$ be the inputs for $h$ (which are $\vee$-gates or literals) from value $(h, \pi)=\top$ we have value $\left(h_{i}, \pi\right)=\top$ so that $\tau\left(h_{i}, \pi\right)=$ Out (if $h_{i}$ is an $\vee$-gate) and $\tau(\neg y, \pi)=O u t$ if $h_{i}$ is the literal $y$ providing the input to $h$ in $T_{\varphi}$ (so that $\neg y$ is the input to $h$ in $H_{\varphi}$ ). It follows that since all input nodes are labelled $O u t, \tau(h, \pi)=I n$. If $h$ is an $\vee$-gate, then from 
$\operatorname{value}(h, \pi)=\top$ at least one of its inputs must have $\operatorname{value}\left(h_{i}, \pi\right)=\top$ giving $\tau\left(h_{i}, \pi\right)=$ In (by induction). But now we obtain $\tau(h, \pi)=O u t$ as claimed.

Finally if $\operatorname{value}(h, \pi)=\perp$ and $h$ is an $\wedge$-gate, we have value $\left(h_{i}, \pi\right)=\perp$ for at least one input $h_{i}$ : if this input is an $\vee$-gate we obtain $\tau\left(h_{i}, \pi\right)=\operatorname{In}$ so that $\tau(h, \pi)=$ Out. If this input is the literal $y$ (in $T_{\varphi}$ ) then $\neg y$ (the input to $h$ in $\left.H_{\varphi}\right)$ has value $(\neg y, \pi)=\top$ so that $\tau(\neg y, \pi)=$ In and $\tau(h, \pi)=$ Out. On the other hand if $h$ is an $\vee$-gate with $\operatorname{value}(h, \pi)=\perp$ then all inputs $h_{i}$ must have value $\left(h_{i}, \pi\right)=\perp$ : these are either literals $y$ (so that value $(y, \pi)=\perp$ giving $\tau(y, \pi)=O u t$ ) or $\wedge$-gates which (by the inductive hypothesis) have $\tau\left(h_{i}, \pi\right)=$ Out: in summary if value $(h, \pi)=\perp$ and $h$ is an $\vee$-gate then each input has $\tau\left(h_{i}, \pi\right)=$ Out so that $\tau(h, \pi)=I n$ as claimed.

\section{Upper bounds on Term Equivalence}

The correspondence between admissible sets of vertices in the formula graph $H_{\varphi}\left(V_{\varphi}, F_{\varphi}\right)$ and partial assignments $\pi$, established over Thms. 1, 2, is not quite strong enough to allow the derivation of our upper bound on TE. By making a final modification to the structure of a formula graph we can, however, obtain the claimed upper bound by exploiting a correspondence between satisfying assignments, $\alpha$ (in the case of $\wedge$-formulae) and the maximal ideal set in the modified graph. We note that, without loss of generality, it may be assumed that that $\varphi\left(X_{n}\right)$ is an $\wedge$-formula: if $\varphi\left(X_{n}\right)$ is an $\vee$-formula simply apply De Morgan's Laws to transform the $\neg \varphi\left(X_{n}\right)$-formula into an $\wedge$-formula noting that $\varphi\left(X_{n}\right) \equiv \vee_{y \in S} S$ if and only if $\neg \varphi\left(X_{n}\right) \equiv \wedge_{y \in S}(\neg y)$.

The graph-theoretic structure considered in the upper bound is now introduced.

Definition 3. Let $\varphi\left(X_{n}\right)$ be an $\wedge$-formula and $H_{\varphi}\left(V_{\varphi}, F_{\varphi}\right)$ its associated formula graph with $g_{m}$ the $\wedge$-gate vertex corresponding to the root of the tree representation 
of $\varphi$. The ideal graph of $\varphi$, denoted $R_{\varphi}$, has vertex set $W_{\varphi}=V_{\varphi} \cup\left\{y_{1}, y_{2}, C, b\right\}$ (where $\left\{y_{1}, y_{2}, C, b\right\}$ are new vertices not occuring in $V_{\varphi}$ ) and edges $F_{\varphi} \cup E_{\varphi}$ where $E_{\varphi}$ contains

$$
\begin{aligned}
& \left\{\left\langle y_{1}, y_{2}\right\rangle,\left\langle y_{2}, y_{1}\right\rangle,\left\langle y_{1}, C\right\rangle,\left\langle y_{2}, C\right\rangle\right\} \\
\cup & \left\{\left\langle C, g_{m}\right\rangle,\left\langle g_{m}, C\right\rangle,\langle C, b\rangle,\langle b, b\rangle\right\} \\
\cup & \left\{\left\langle b, x_{i}\right\rangle: 1 \leq i \leq n\right\} \cup\left\{\left\langle b, \neg x_{i}\right\rangle: 1 \leq i \leq n\right\}
\end{aligned}
$$

The following characterisation of ideal sets and conditions for a vertex to be a member of the maximal ideal set are stated in [7]. ${ }^{4}$

\section{Fact 2.}

a. Let $H(V, F)$ be any directed graph and $S$ a subset of $V$. The set $S$ is an ideal set if and only if both of the following hold:

P1. $S$ is an admissible set of $H(V, F)$.

P2. For all $w \in S^{-}$, there is no admissible set of $H(V, F)$ containing $w$.

b. Let $\mathcal{M}$ be the (unique) maximal ideal set of $H(V, F)$ and $v$ a vertex from $V$.

Then $v \in \mathcal{M}$ if and only if both of the following hold:

M1. For all $w \in\{v\}^{-}$there is no admissible set of $H(V, F)$ that contains $w$.

M2. For all $w \in\{v\}^{-}, \mathcal{M} \cap\{w\}^{-} \neq \emptyset$, i.e. $\mathcal{M}$ contains at least one vertex from $\{w\}^{-}$.

The key property of ideal graphs we need is

Theorem 3. Let $\varphi\left(X_{n}\right)$ be an $\wedge$-formula for which $\varphi\left(X_{n}\right) \not \equiv \perp$ and $\varphi\left(X_{n}\right) \not \equiv \top$, i.e. $\varphi\left(X_{n}\right)$ is satisfiable and not a tautology. ${ }^{5}$ Let $R_{\varphi}\left(W_{\varphi}, F_{\varphi} \cup E_{\varphi}\right)$ be the ideal

\footnotetext{
${ }^{4}$ The full proofs of these may be found in [8].

${ }^{5}$ Note that if $\varphi\left(X_{n}\right)=\psi_{1}\left(X_{n}\right) \wedge \psi_{2}\left(X_{n}\right)$ then $\varphi\left(X_{n}\right)$ will be a tautology if both $\psi_{1}$ and $\psi_{2}$ are tautologies.
} 
graph constructed from $\varphi\left(X_{n}\right)$ and $\mathcal{M}_{\varphi} \subseteq W_{\varphi}$ be the maximal ideal set of $R_{\varphi}$.

$$
\mathcal{M}_{\varphi} \neq \emptyset \text { if and only if } \varphi\left(X_{n}\right) \in \mathrm{TE}
$$

Proof: Suppose $\mathcal{M}_{\varphi} \neq \emptyset$. From Fact 2(a) we have $\mathcal{M}_{\varphi} \cap\left\{y_{1}, y_{2}, C\right\}=\emptyset$ : the sets $\left\{y_{1}\right\}$ and $\left\{y_{2}\right\}$ are both admissible, however, $y_{1} \in\left\{y_{2}\right\}^{-}, y_{2} \in\left\{y_{1}\right\}^{-}$ and both vertices are in $\{C\}^{-}$. The vertex $b$ is not in any admissible set since $\langle b, b\rangle \in F_{\varphi} \cup E_{\varphi}$. In total we deduce from $\mathcal{M}_{\varphi} \neq \emptyset$ that $\mathcal{M} \subseteq V_{\varphi}$ - the set of vertices defining the formula graph, $H_{\varphi}$, of $\varphi$.

We now argue that $\mathcal{M}_{\varphi} \neq \emptyset$ implies $g_{m} \in \mathcal{M}_{\varphi}$ (recall that $g_{m}$ is the $\wedge$-gate in $V_{\varphi}$ corresponding to the root of the tree representation, $\left.T_{\varphi}\right)$.

For suppose this were not so. From $g_{m} \notin \mathcal{M}_{\varphi}$ and Fact 2(b)

$$
\mathcal{M}_{\varphi} \cap\left\{x_{1}, \ldots, x_{n}, \neg x_{1}, \ldots \neg x_{n}\right\}=\emptyset
$$

That is, no literal vertex could belong to $\mathcal{M}_{\varphi}$ : the vertex $b$ is in $\{x\}^{-}$hence were any literal vertex to be in $\mathcal{M}_{\varphi}$ this would force $g_{m} \in \mathcal{M}_{\varphi}$ since $\{b\}^{-}=\left\{b, g_{m}\right\}$. It is now easy to see that $g_{m} \notin \mathcal{M}_{\varphi}$ forces $\mathcal{M}_{\varphi}=\emptyset$ : we have already argued that $\mathcal{M}_{\varphi}$ must be a subset of $V_{\varphi}$, thus, from the assumption that $g_{m} \notin \mathcal{M}_{\varphi}$ and the consequence of $\mathcal{M}_{\varphi}$ containing no literal vertices, if $\mathcal{M}_{\varphi} \neq \emptyset$ then it can only contain gate vertices. Consider any gate vertex $h$. If $h$ has a literal, $y$, as an input then $h \notin \mathcal{M}_{\varphi}$ from Fact 2(b): the only choices from $\{y\}^{-}$being $\neg y$ and $b$ neither of which belong to $\mathcal{M}_{\varphi}$. If $h$ has only gate vertices $\left\{h_{1}, \ldots, h_{r}\right\}$ as inputs then $\mathcal{M}_{\varphi} \cap\left\{h_{j}\right\}^{-}$has to be non-empty: now repeating the same argument (with respect to vertices $\left\{h_{j}\right\}^{-}$) we eventually reach the position that some literal vertex must belong to $\mathcal{M}_{\varphi}$. We deduce that if $\mathcal{M}_{\varphi} \neq \emptyset$ then $g_{m} \in \mathcal{M}_{\varphi}$.

From $g_{m} \in \mathcal{M}_{\varphi}$ and our analysis above it follows that

$$
\mathcal{M}_{\varphi} \cap\left\{x_{1}, \ldots, x_{n}, \neg x_{1}, \ldots \neg x_{n}\right\} \neq \emptyset
$$


Let $S$ be the set of literal vertices occurring in $\mathcal{M}_{\varphi}$. First notice that $S$ cannot contain both a literal and its negation since $\mathcal{M}_{\varphi}$ must be conflict-free. Without loss of generality let $S=\left\{x_{1}, \ldots, x_{k}\right\}$. We now claim that $\varphi\left(X_{n}\right)=\wedge_{i=1}^{k} x_{i}$ so establishing that $\varphi\left(X_{n}\right)$ is a positive instance of TE.

Now $S \cup\left\{g_{m}\right\} \subseteq \mathcal{M}_{\varphi}$ indicates (by the definition of ideal set) that $S \cup\left\{g_{m}\right\}$ is a subset of every preferred set of $R_{\varphi}$. Any such preferred set consists of some subset, $Q$, of vertices from $V_{\varphi}$ and exactly one of the vertices from $\left\{y_{1}, y_{2}\right\}$ so that from Thm. 1(b), $Q=\operatorname{In}_{\varphi}(\alpha)$ for some satisfying ${ }^{6}$ assignment $\alpha$ of $\varphi\left(X_{n}\right)$. It follows that every assignment in which $x_{i}:=\top(1 \leq i \leq k)$ will satisfy $\varphi\left(X_{n}\right)$ regardless of how the variables $X_{n} \backslash\left\{x_{1}, \ldots, x_{k}\right\}$ are assigned. In addition, however, no assignment in which $x_{i}:=\perp$ (for $1 \leq i \leq k$ ) can satisfy $\varphi\left(X_{n}\right)$. For suppose there were a satisfying assignment, $\beta$ with $x_{1}=\perp$, then in this case both $\operatorname{In}_{\varphi}(\beta) \cup\left\{y_{1}\right\}$ and $\operatorname{In}_{\varphi}(\beta) \cup\left\{y_{2}\right\}$ would be preferred sets of $R_{\varphi}$ : neither of these, however, contain $x_{1}$, thereby contradicting $x_{1} \in \mathcal{M}$. In summary, $x_{i}:=\top$ for each $1 \leq i \leq k$ satisfies $\varphi\left(X_{n}\right)$ but $x_{i}:=\perp$ for any $1 \leq i \leq k$ fails to satisfy $\varphi\left(X_{n}\right)$, i.e. $\varphi\left(X_{n}\right) \equiv \wedge_{i=1}^{k} x_{i}$ as required.

For the converse direction, suppose that $\varphi\left(X_{n}\right) \equiv \wedge_{y \in S} y$ for some subset, $S$, of literals over $X_{n}$. Without loss of generality, assume that $S=\left\{x_{1}, \ldots, x_{k}\right\}$. We show that $\left\{g_{m}\right\} \cup S=\left\{g_{m}, x_{1}, \ldots, x_{k}\right\} \subset W_{\varphi}$ is a subset of $\mathcal{M}_{\varphi}$. From the structure of $R_{\varphi}$ it follows that neither $\left\{y_{1}\right\}$ nor $\left\{y_{2}\right\}$ are preferred sets of $R_{\varphi}$ (since $\{y\} \cup I n_{\varphi}(\alpha)$ is admissible when $\alpha$ satisfies $\varphi$ for $y \in\left\{y_{1}, y_{2}\right\}$ ). Thus the preferred sets of $R_{\varphi}$ are of the form $\left\{y_{1}\right\} \cup I n_{\varphi}(\alpha)$ and $\left\{y_{2}\right\} \cup I n_{\varphi}(\alpha)$ for satisfying assignments $\alpha$ of $\varphi$. The only such assignments, however, select $x_{i}(1 \leq i \leq k)$ as literal vertices. It follows that every preferred set of $R_{\varphi}$ contains $\left\{g_{m}, x_{1}, \ldots, x_{k}\right\}$ as a subset. Furthermore, using the partial assignment $\pi$ in which $p_{i}=\top$ (for

\footnotetext{
${ }^{6}$ Satisfying since $g_{m} \in \operatorname{In} n_{\varphi}(\alpha)$ so that value $\left(g_{m}, \alpha\right)=\top$ from Thm. 2 .
} 
$1 \leq i \leq k)$ and $p_{i}=*$ for all other variables we deduce from $\varphi\left(X_{n}\right) \equiv \wedge_{i=1}^{k} x_{i}$ that $\operatorname{In}_{\varphi}(\pi)$ is admissible via Thm. 1(a) ${ }^{7}$ and a subset of every preferred set of $R_{\varphi}$. In other words $\operatorname{In}_{\varphi}(\pi)$ is an ideal set and $\mathcal{M}_{\varphi} \neq \emptyset$ as claimed.

An immediate corollary of Thm. 3 is that Algorithm 1, below, will construct a witnessing subset, $S$, of literals such that $\varphi\left(X_{n}\right) \equiv \wedge_{y \in S} y$ if such a subset exists:

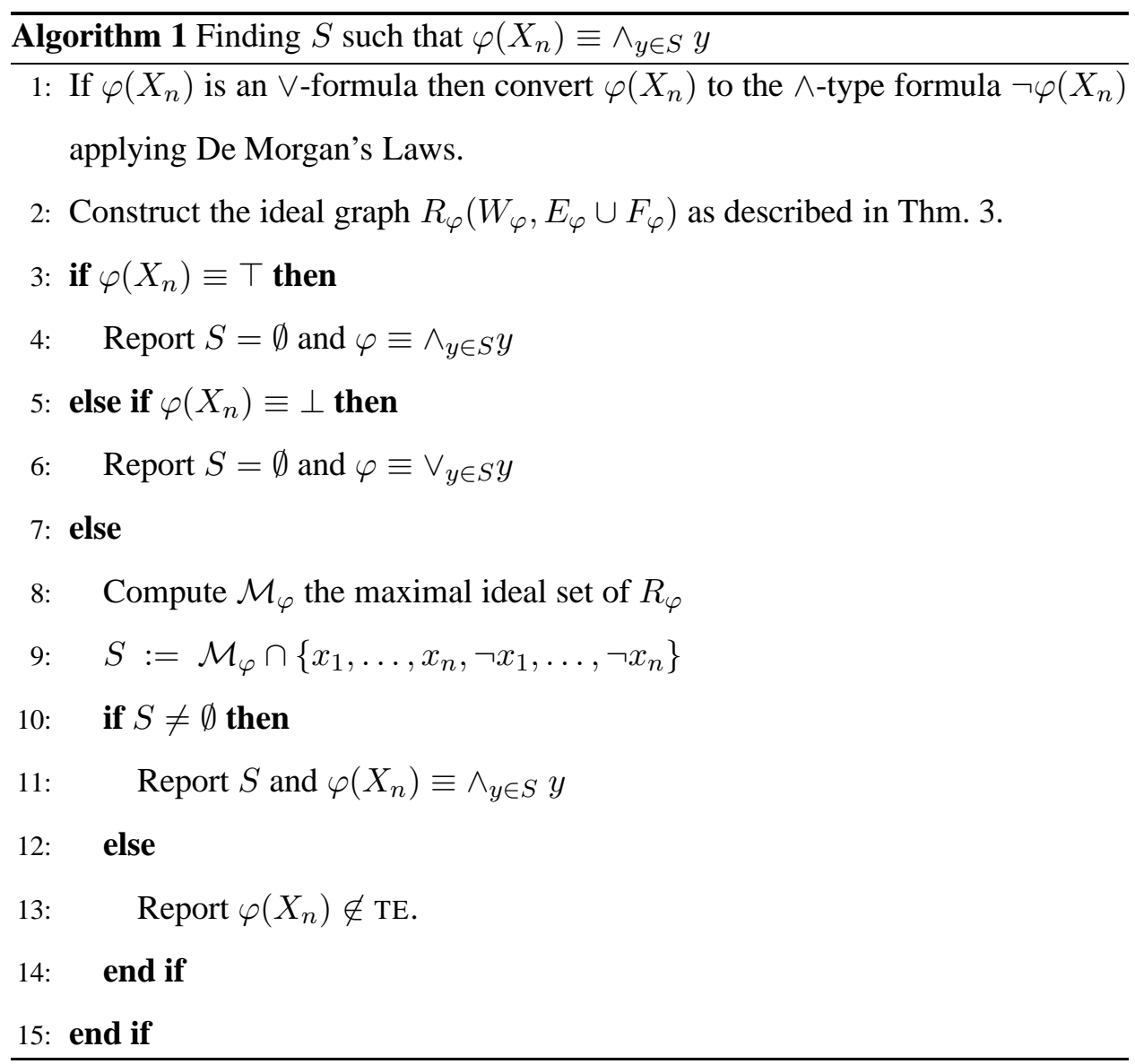

The correctness of Algorithm 1 is immediate from Thm. 3. All that is needed for the claimed upper bound on FTE is to show that the maximal ideal set can be

\footnotetext{
${ }^{7}$ Note that $C \in\left\{\operatorname{In}_{\varphi}(\pi)\right\}^{-}$is countered by $g_{m} \in\{C\}^{-}$.
} 
computed in $\mathrm{FP}_{\|}^{\mathrm{NP}}$. As we noted in Fact 1(h) such an upper bound has been given in Dunne [7,8]. For completeness we outline this algorithm (as specialised to the particular instance $R_{\varphi}$ ).

Theorem 4. Algorithm 1 can be implemented in $\mathrm{FP}_{\|}^{\mathrm{NP}}$.

Proof: Given $\varphi\left(X_{n}\right)$ which we assume to be an $\wedge$-formula it is clear that the ideal graph $R_{\varphi}\left(W_{\varphi}, F_{\varphi} \cup E_{\varphi}\right)$ may be constructed in polynomial time (in the size of $\varphi$ ). After checking if $\varphi\left(X_{n}\right) \equiv \top$ or $\varphi\left(X_{n}\right) \equiv \perp$ construct the following partition of $W_{\varphi}:$

$$
\begin{aligned}
& W_{\mathrm{REJ}}=\left\{v \in W_{\varphi}: v \text { is not in any admissible set of } R_{\varphi}\right\} \\
& W_{\mathrm{POS}}=\left\{v \in W_{\varphi}:\{v\}^{-} \cup\{v\}^{+} \subseteq W_{\mathrm{REJ}}\right\} \backslash W_{\mathrm{REJ}}
\end{aligned}
$$

Now form the bipartite graph $B\left(W_{\mathrm{POS}}, W_{\mathrm{REJ}}, D\right)$ in which

$$
D=F_{\varphi} \backslash\left\{\langle v, w\rangle: v \in W \backslash W_{\mathrm{POS}} \text { and } w \in W \backslash W_{\mathrm{POS}}\right\}
$$

It is shown in Dunne [7] that $\mathcal{M}_{\varphi}$ is the maximal admissible subset of $W_{\text {POS }}$ in this bipartite graph. The algorithm below, from Dunne [6] computes this set in polynomial time.

To complete the upper bound proof it suffices to observe that constructing this bipartite graph requires only computing the set $W_{\text {REJ }}$ which (in conjunction with testing $\varphi\left(X_{n}\right) \equiv \top$ or $\left.\varphi\left(X_{n}\right) \equiv \perp\right)$ can be done using $\left|W_{\varphi}\right|+2$ parallel calls to an NP oracle: 2 calls to determine $\varphi\left(X_{n}\right) \equiv \top$ or $\varphi\left(X_{n}\right) \equiv \perp$; and a further $\left|W_{\varphi}\right|$ calls to detemrine which vertices of $R_{\varphi}$ are members of some admissible set.

Corollary 1. TE $\in \mathrm{P}_{\|}^{\mathrm{NP}}$

Proof: Immediate from the Thm. 4. 


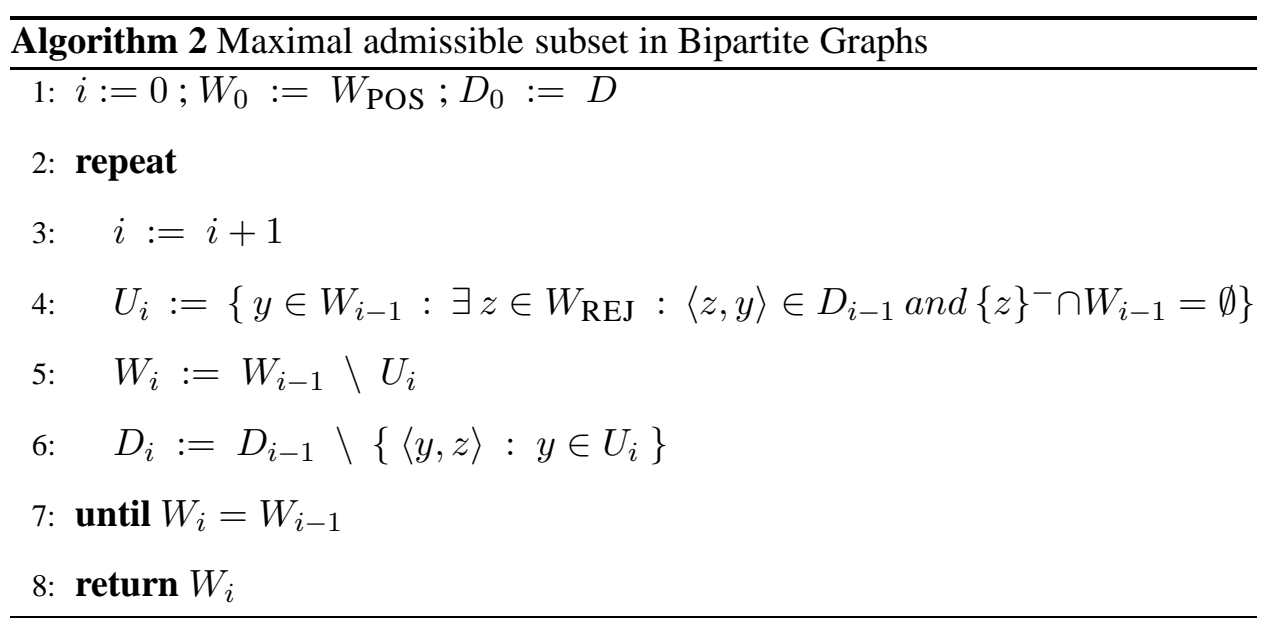

\section{Conclusion}

In this note we have considered a variant of the Minimal Expression Equivalence problem (MEE) studied by Hemaspaandra and Wechsung [11, 12] whereby the form of witnessign smaller formulae is restricted to elementary conjunctions

and disjunctions. Our main result shows this variant can be decided in $\mathrm{P}_{\|}^{\mathrm{NP}}$ (with the search form belonging to the analogous function complexity class). This class has been shown to provide a lower bound for the general MEE problem. One feature of interest in our proof is the range of technical material originating from recent work on extension-based semantics in argument systems, in particular the correspondence between ideal sets and witnessing subsets of literals in the so-called ideal graph derived from a given formula. One of the original applications of argument system semantics was in modelling problems in non-classical logics (as opposed to the propositional basis of the current article). It would, therefore, be of some interest to see to what extent further interaction between argumentation semantics and algorithms for deciding properties of propositional formulae is possible. 


\section{References}

[1] T. J. M. Bench-Capon and P. E. Dunne. Argumentation in artificial intelligence. Artificial Intelligence, 171:619-641, 2007.

[2] Y. Dimopoulos and A. Torres. Graph theoretical structures in logic programs and default theories. Theoretical Computer Science, 170:209-244, 1996.

[3] P. M. Dung. On the acceptability of arguments and its fundamental role in nonmonotonic reasoning, logic programming, and $N$-person games. Artificial Intelligence, 77:321-357, 1995.

[4] P. M. Dung, P. Mancarella, and F. Toni. Computing ideal sceptical argumentation. Artificial Intelligence, 171:642-674, 2007.

[5] P. E. Dunne. The complexity of Boolean networks Academic Press, 1988

[6] P. E. Dunne. Computational properties of argument systems satisfying graphtheoretic constraints. Artificial Intelligence, 171:701-729, 2007.

[7] P. E. Dunne. The computational complexity of ideal semantics I: abstract argumentation frameworks. In Proc. 2nd Int. Conf. on Computational Models of Argument, volume 172 of FAIA, pages 147-158. IOS Press, 2008.

[8] P. E. Dunne. The computational complexity of ideal semantics. Tech. Report ULCS-08-015, Dept. of Computer Science, Univ. of Liverpool, 2008 (submitted)

[9] P. E. Dunne and T. J. M. Bench-Capon. Coherence in finite argument systems. Artificial Intelligence, 141:187-203, 2002. 
[10] P. E. Dunne and M. Wooldridge. Complexity of abstract argumentation. In I. Rahwan and G. Simari, editors, Argumentation in AI. Springer-Verlag, 2009. (in press).

[11] E. Hemaspaandra and G. Wechsung The Minimization Problem for Boolean Formulas. In: Proc. 38th Annual Symp. on Foundations of Computer Science (FOCS'97), page 575-584, 1997

[12] E. Hemaspaandra and G. Wechsung The Minimization Problem for Boolean Formulas. SIAM J. Comput., 31(6):1948-1958, 2002 